\title{
Origin of ferroelectric polarization in tetragonal tungsten-bronze-type oxides
}

\author{
Gerhard Henning Olsen, ${ }^{1,2}$ Ulrich Aschauer, ${ }^{2,3}$ Nicola A. Spaldin, ${ }^{2}$ Sverre Magnus Selbach, ${ }^{1}$ and Tor Grande ${ }^{1, *}$ \\ ${ }^{1}$ NTNU Norwegian University of Science and Technology, \\ Department of Materials Science and Engineering, NO-7491 Trondheim \\ ${ }^{2}$ ETH Zurich, Materials Theory, Wolfgang-Pauli-Strasse 27, CH-8093 Zurich \\ ${ }^{3}$ University of Bern, Department of Chemistry and Biochemistry, Freiestrasse 3, CH-3012 Bern
}

(Dated: March 19, 2016)

\begin{abstract}
The origin of ferroelectric polarization in the tetragonal tungsten-bronze-type oxide strontium barium niobate (SBN) is investigated using first-principles density functional calculations. We study in particular the relationship between the polarization and the cation and vacancy ordering on the alkali earth lattice sites. Lattice dynamical calculations for paraelectric structures demonstrate that all cation configurations that can be accommodated in a $1 \times 1 \times 2$ supercell result in a single unstable polar phonon, composed primarily of relative $\mathrm{Nb}-\mathrm{O}$ displacements along the polar axis, as their dominant instability. The majority of the configurations also have a second octahedral tilt-mode instability which couples weakly to the polar mode. The existence of the tilt mode is strongly dependent on the local cation ordering, consistent with the fact that it is not found experimentally. Our results suggest that ferroelectricity in the SBN system is driven by a conventional second-order Jahn-Teller mechanism caused by the $d^{0} \mathrm{Nb}^{5+}$ cations, and demonstrate the strong influence of the size of $\mathrm{Sr}$ and $\mathrm{Ba}$ on the lattice distortions associated with polarization and octahedral tilting. Finally, we suggest a mechanism for the relaxor behavior in Sr-rich SBN based on Sr displacement inside pentagonal channels in the TTB structure.
\end{abstract}

PACS numbers: 71.15.Mb, 77.80.Jk, 77.80.bg

Ferroelectric oxides with tetragonal tungsten-bronze (TTB) structure have been known since 1953 [3], and their polar properties are both widely studied and used in applications. One of the simplest TTB compounds, lead metaniobate $\left(\mathrm{Pb}_{5} \mathrm{Nb}_{10} \mathrm{O}_{30}\right.$ or PN), is commercially available and attractive for hightemperature piezoelectric sensor applications. In spite of their popularity, however, a fundamental explanation of the mechanism leading to spontaneous polarization in TTBs has to our knowledge not been reported. One possible origin is $\mathrm{Nb}^{5+}$, which is a ferroelectrically active cation in perovskites owing to a second-order Jahn-Teller mechanism enabled by its $d^{0}$ electron configuration $[4,5]$. Fundamental studies on TTBs are challenging, partly due to multiple competing phases: PN, for example, is at ambient temperature metastable in the TTB structure with respect to a rhombohedral polymorph [6-8]. Most other tungsten-bronze oxides are solid solutions with structural and chemical disorder, consistent with many of them being relaxor-type ferroelectrics [9-11].

The aristotype unit cell with the general formula $(\mathrm{A} 1)_{2}(\mathrm{~A} 2)_{4} \mathrm{C}_{4}(\mathrm{~B} 1)_{2}(\mathrm{~B} 2)_{8} \mathrm{O}_{30}$ has six sites available for A-type cations and ten for B-type. The structure is highly anisotropic, with the A1 and A2 sites forming channels along the $c$ direction. The A1 sites are formally 12-coordinated and similar to the A sites in perovskites, while the A2 sites are pentagonal and formally 15-coordinated. The B1 and B2 sites are octahedrally coordinated, with corner-sharing $\mathrm{BO}_{6}$ octahedra forming the structural framework. Many niobates and tantalates crystallize as TTBs where $\mathrm{Nb}$ or Ta resides on the octahedral B sites. The A sites can accommodate a wide range of cations, from alkali and alkaline earth metals to $p$ block cations such as $\mathrm{Pb}^{2+}$ and $\mathrm{Bi}^{3+}$. The triangular, 9-coordinated $\mathrm{C}$ sites can only accommodate small cations such as $\mathrm{Li}^{+}$and $\mathrm{Nb}^{5+}$ and are therefore often completely vacant, while the A sites can be partially or completely filled [12-14]. Due to this flexibility, disorder in
TTBs takes place both through site interchange and partial occupancy.

We choose the simple $\left(\mathrm{Sr}_{x} \mathrm{Ba}_{1-x}\right)_{5} \mathrm{Nb}_{10} \mathrm{O}_{30}(\mathrm{SBN})$ as a model system for investigating the ferroelectric polarization in TTBs. The divalent cations $\mathrm{Sr}^{2+}$ and $\mathrm{Ba}^{2+}$ occupy the A1 and $\mathrm{A} 2$ sites, with one of the six total A sites unoccupied to maintain electroneutrality [12]. Only intermediate SBN compositions $(0.25<x<0.75)$ are accessible by conventional synthesis methods, while the end members are metastable with respect to other more stable polymorphs $[15,16]$. SBN materials with TTB structure have attractive electro-optical properties such as high second harmonic yield and good pyroelectric performance [2], and they are also of interest as lead-free ferroelectrics $[17,18]$. Ba-rich SBN compositions are classical ferroelectrics with a sharp dielectric maximum at $T_{\mathrm{C}}$ and Curie-Weiss behavior in the paraelectric state, while Sr-rich compositions are relaxor ferroelectrics with a broad dielectric maximum and a strong frequency dispersion in dielectric permittivity [9]. The transition is gradual, but relaxor behavior dominates for compositions with $x>0.50[2,9,11]$. The mechanism triggering this crossover is not known, although a recent study found relaxor behavior in TTBs to correlate with the existence of incommensurate superstructures [11]. Here, we present first-principles calculations of the stability and lattice dynamics of the end-point compounds of SBN, $\mathrm{Ba}_{5} \mathrm{Nb}_{0} \mathrm{O}_{30}(\mathrm{BN})$ and $\mathrm{Sr}_{5} \mathrm{Nb}_{10} \mathrm{O}_{30}(\mathrm{SN})$. We study ten different cation-vacancy configurations, and in each case calculate the structural ground state and its associated spontaneous polarization. Based on the behavior of the end members, we discuss how the $\mathrm{Sr} / \mathrm{Ba}$ ratio affects the properties of SBN, with particular focus on the ferroelectric mechanism and how it is influenced by different A-site cation size.

Structural data for BN and SN with TTB structure were taken from our previous work [19] where we calculated the 

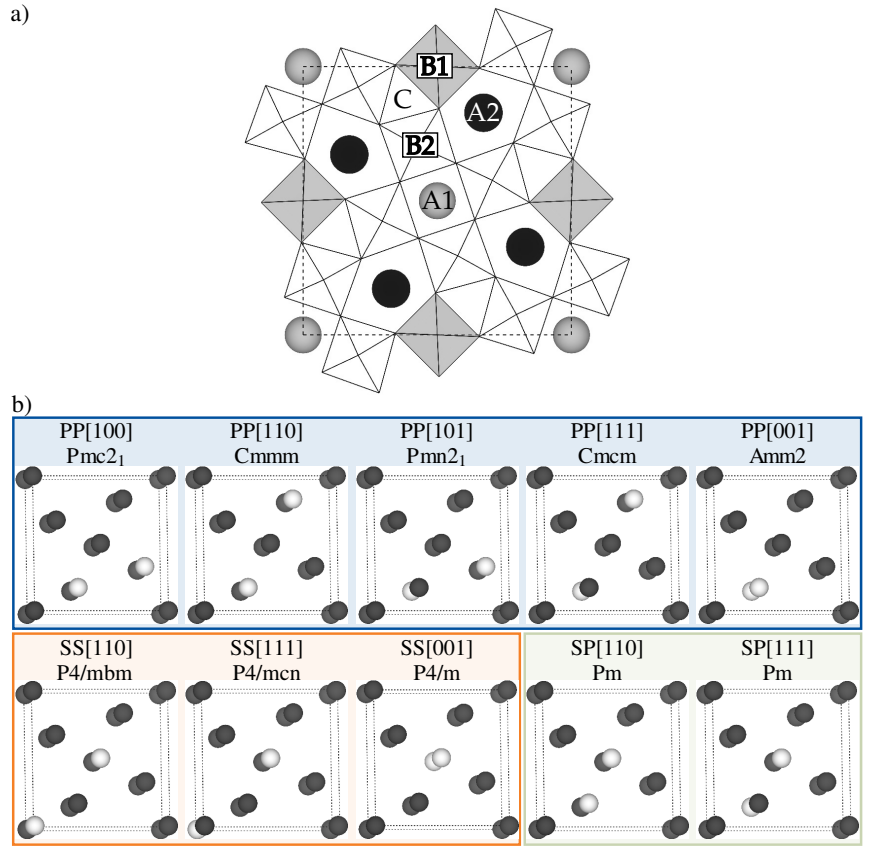

Figure 1. (Color online) a) Projection along $c$ of the aristotype tetragonal tungsten-bronze (TTB) unit cell with space group symmetry $P 4 / m b m$ and general formula $(\mathrm{A} 1)_{2}(\mathrm{~A} 2)_{4} \mathrm{C}_{4}(\mathrm{~B} 1)_{2}(\mathrm{~B} 2)_{8} \mathrm{O}_{30}$. b) The ten cation configurations possible for a $1 \times 1 \times 2$ supercell. Each entry gives the systematic name, local space group symmetry and a sketch of the configuration (cations grey, vacancies white). Boxes separate orthorhombic (PP), tetragonal (SS) and monoclinic (SP) configurations. Figures created with VESTA [1].

high-symmetry paraelectric structures for the ten distinct cation configurations that can be accommodated in a $1 \times 1 \times 2$ supercell of the aristotype unit cell (Figure 1a). The configurations are shown in Figure 1b, along with their space group symmetries [20] and a label indicating the location of the vacancies. The nomenclature describes whether the two vacancies are located on pentagonal A2 sites (P) or on square A1 sites (S), as well as the approximate vector between the vacancies ([100] etc.) [19]. Vacancy ordering lowers the symmetry from the aristotype space group $P 4 / m b m$ to either tetragonal (SS configurations), orthorhombic (PP) or monoclinic (SP) space group symmetries (Figure 1b).

Starting from these ten paraelectric structures, we calculated the phonon instabilities and the fully relaxed ground states using density functional theory as implemented in the VASP code [21]. We used the gradient-corrected PBEsol functional [22] to describe the exchange-correlation energy and the projector-augmented wave method [23] with standard VASP potentials and valence electron configurations $4 s^{2} 4 p^{6} 5 s^{2}$ for $\mathrm{Sr}, 5 s^{2} 5 p^{6} 6 s^{2}$ for $\mathrm{Ba}, 4 s^{2} 4 p^{6} 4 d^{3} 5 s^{2}$ for $\mathrm{Nb}$ and $2 s^{2} 2 p^{4}$ for O. Wave functions were expanded in plane waves up to an energy cutoff of $550 \mathrm{eV}$, and reciprocal space integrals were done on a $2 \times 2 \times 3$ Monkhorst-Pack grid [24] for the 90-atom supercells. All structures were relaxed until the forces on all ions converged to below $10^{-4} \mathrm{eV} \AA^{-1}$. Phonon calculations
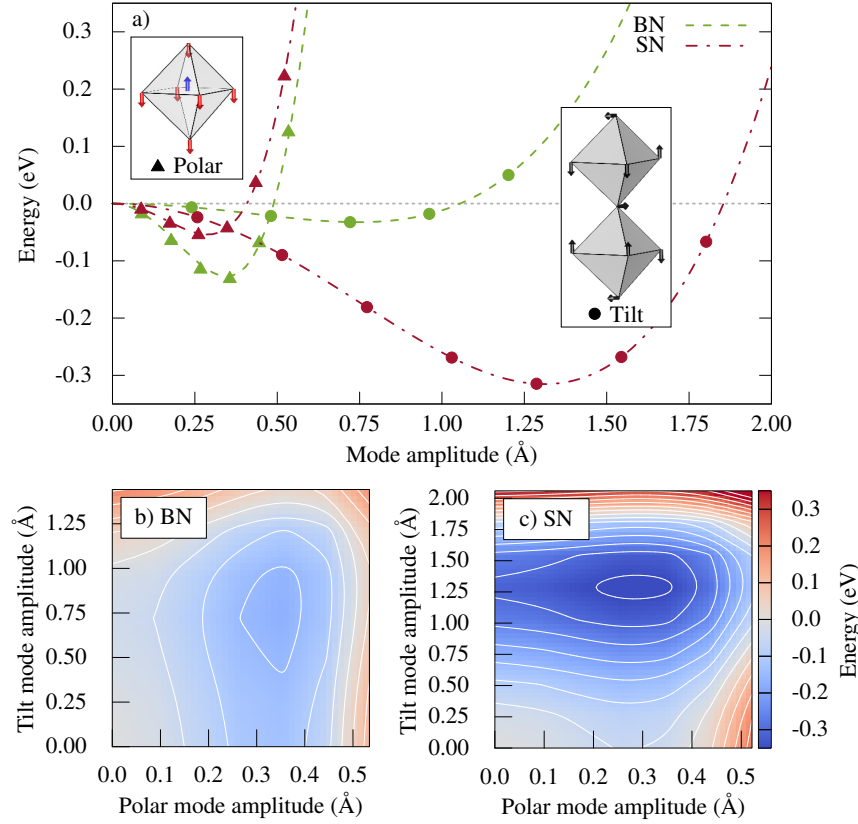

Figure 2. (Color online) a) Potential energy curves for $\mathrm{Ba}_{5} \mathrm{Nb}_{10} \mathrm{O}_{30}$ (BN) and $\mathrm{Sr}_{5} \mathrm{Nb}_{10} \mathrm{O}_{30}(\mathrm{SN})$ in the $\mathrm{SS}[001]$ configuration, showing energy lowering from freezing in polar and tilt-mode instabilities independently from the paraelectric structure. Points show calculated energies, lines are polynomial fits to $\Delta E=\alpha Q^{2}+\beta Q^{4}$ where $Q$ is the mode amplitude $\left(Q=1 \AA\right.$ means that $Q^{2}=\sum_{i} u_{i}^{2}=1 \AA^{2}$ where $u_{i}$ is the distortion of atom $i$ and the sum is over all atoms in a unit cell). Insets show the structural distortions associated with the phonons. b) and c) Contour plots for BN and SN, respectively, showing the weak coupling between the polarization and tilt modes (SS[001] configuration).

were performed with the finite displacement method as implemented in the PHONOPY code $[25,26]$, with displacements of $0.01 \AA$ used for calculation of the force constants. Spontaneous polarization in periodic structures was calculated using the Berry phase method [27-29], and Born effective charges (BECs) were obtained using the linear response routines in VASP.

Phonon calculations for the paraelectric structures reveal imaginary frequency phonons, corresponding to structural instabilities, for all ten configurations of both BN and SN. The phonon with the highest imaginary frequency is in all cases a polar mode with $\Gamma^{-}$symmetry, corresponding to the displacement pattern illustrated in the left inset of Figure 2a. The mode consists primarily of a cooperative displacement along the tetragonal $c$ direction of all $\mathrm{Nb}$ ions relative to the surrounding oxygens in the $\mathrm{NbO}_{6}$ octahedra. This is a polar mode, similar to the soft mode responsible for the ferroelectric transition in perovskite ferroelectrics [30], and produces a polarization along $c$. Consistent with the imaginary frequency of the mode, we find that displacing the atoms according to the eigenvector of the polar mode (we refer to this as "freezing in" the mode in what follows) lowers the energy in all cases. The energy lowering as a function of mode amplitude [31] is shown in 


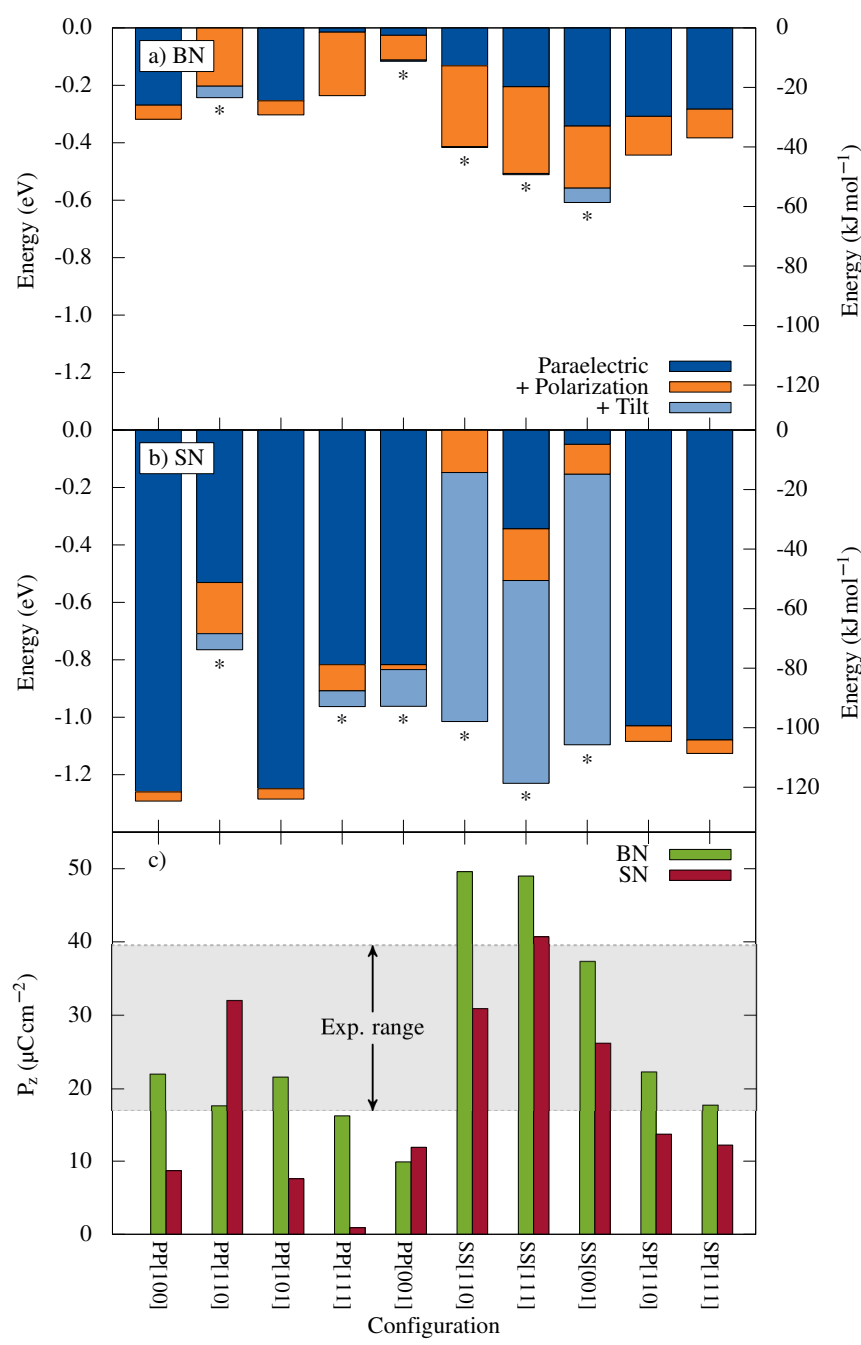

Figure 3. (Color online) Energy landscape for a) $\mathrm{Ba}_{5} \mathrm{Nb}_{10} \mathrm{O}_{30}(\mathrm{BN})$ and b) $\mathrm{Sr}_{5} \mathrm{Nb}_{10} \mathrm{O}_{30}(\mathrm{SN})$, showing the stepwise energy lowering from freezing in the unstable phonons in sequence. All configurations have a polar instability ("Polarization"), while 11 of 20 (marked with*) have a secondary tilt-mode instability ("Tilt") after freezing in the polar mode. Energies are per 45-atom unit cell, with the zero level set to the highest-energy paraelectric structure ("Paraelectric"). c) Spontaneous polarization for $\mathrm{BN}$ and $\mathrm{SN}$ in their fully relaxed ground states. $P_{z}$ is the polarization along the tetragonal $c$ direction. Shaded region marks values reported by Glass [2].

Figure $2 \mathrm{a}$ for $\mathrm{BN}$ and $\mathrm{SN}$ in the SS[001] configuration. A non-polar mode consisting of octahedral tilting around axes in the $x y$ plane (Figure 2a, right inset) is also present in all cases. This mode has a smaller imaginary frequency than the polar mode for both compounds in all configurations. The energy lowering associated with the tilt mode is significantly larger for $\mathrm{SN}$ than for BN, as shown in Figure 2a. The tilt mode also lowers the energy more than the polar mode for $\mathrm{SN}$, despite having a lower imaginary frequency. Simultaneous freezing-in of the polar and tilt modes (Figure 2b-c) show that the coupling between the modes is weak for both compounds.

We systematically search for the dynamically stable ground

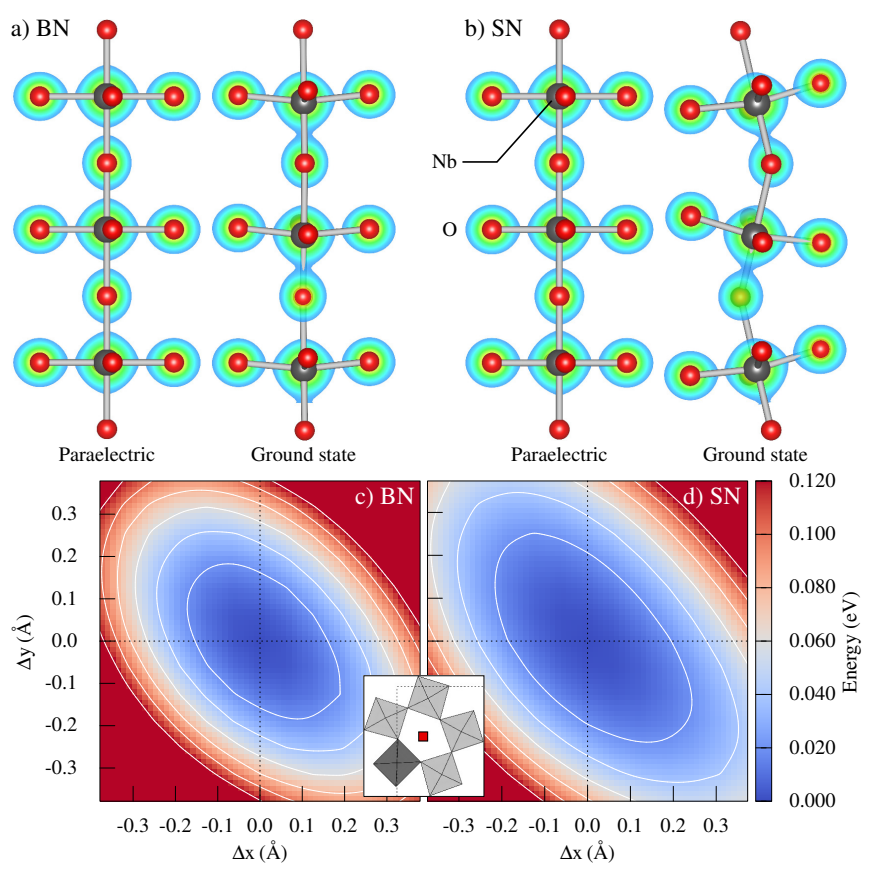

Figure 4. (Color online) a) and b) Charge density plots for $\mathrm{Ba}_{5} \mathrm{Nb}_{10} \mathrm{O}_{30}(\mathrm{BN})$ and $\mathrm{Sr}_{5} \mathrm{Nb}_{10} \mathrm{O}_{30}(\mathrm{SN})$ in paraelectric and ground state structures (isosurface value $0.15 e \AA^{-3}$ ). c) and d) Potential energy surfaces for the $\mathrm{A} 2$-site cation in $\mathrm{BN}$ and $\mathrm{SN}$ in the paraelectric SS[001] configuration (energy per 45-atom unit cell). The reference position of the cation is in the middle of each plot, and the inset shows the location and size of the mapped area (red square).

state of each configuration of both compounds by freezing in the polar mode, which always has the largest imaginary frequency of the unstable modes, at a mode amplitude close to the energetic minimum. We then relax the structures, allowing the volume and cell parameters to change. After repeating the phonon calculations for the relaxed polar structures, no further instabilities are found in 9 of the 20 cases. In the remaining 11 , a second, non-polar instability persists, its eigenvector qualitatively identical to the tilt-mode found in the paraelectric structures. After also freezing in this mode, relaxing the structures and repeating phonon calculations, we obtain the stable ground state structure for both compounds in all configurations. The relative energies of all the configurations are shown in Figure 3a-b. The energy landscape is different for the two compounds: The SS[001] configuration has the lowest ground state energy for $\mathrm{BN}$, while for $\mathrm{SN}$ the lowest energy is obtained for $\mathrm{PP}[100]$. No single configuration has a substantially lower energy than the others, especially for SN, where PP[101] and $\mathrm{SS}[111]$ are particularly close in energy to the lowest-energy configuration. Consistent with the fixed-volume mapping of unstable modes shown in Figure 2, the energy relaxation following the polar distortion is more pronounced for $\mathrm{BN}$, while the tilt mode has a larger impact on the ground state energy for SN. For SN there are large energy differences between the paraelectric structures, but the substantial contribution from tilt-mode distortions reduces these differences. The resulting 
spontaneous polarizations are shown in Figure $3 \mathrm{c}$ and compared to literature data. Several of the paraelectric reference structures have polar space group symmetries (Figure 1b), but none allow a polarization along $z$. We focus here on the polarization component along the tetragonal axis, $P_{z}$, which results from freezing in the unstable polar phonon. For the majority of the configurations, BN has a larger $P_{z}$ compared to the same configuration for SN. 11 of the 20 ground state structures have tilt-mode instabilities, but the polar nature remains in all cases.

We now turn to understanding the chemical bonding which stabilizes the polarization. In Figure $4 a-b$ we show the charge density for the paraelectric and ground state structures for BN and SN in the SS[001] configuration. This configuration is suitable for comparing the effects of polar and tilt-mode instabilities, in addition to being the true zero-kelvin ground state for SN. At the chosen isosurface value, we see a distinct "necking" between $\mathrm{Nb}$ and $\mathrm{O}$ in the direction of the polar distortion, pointing to a covalent bonding between the atoms. As further evidence for the role of $\mathrm{Nb}-\mathrm{O}$ covalency, we calculate BECs [32] for all configurations of both compounds in their paraelectric states (supplementary information). Consistent with the charge density visualization in Figure $4 a-b$, the BECs for $\mathrm{Nb}$ and $\mathrm{O}$ along $c$ are large, exceeding +9 for $\mathrm{Nb}$ and -7 for oxygen, in comparison to their respective formal charges of +5 and -2 . The values are similar to BECs reported for $\mathrm{KNbO}_{3}$ and $\mathrm{NaNbO}_{3}$, following the trend of $Z^{*}$ being proportional to the formal charge of the B-site cation [32, 33]. The variation in $\mathrm{BEC}$ between $\mathrm{BN}$ and $\mathrm{SN}$ is minor, pointing to a similar degree of $\mathrm{Nb}-\mathrm{O}$ covalency in the two compounds, which is also indicated by their similar electronic densities of states (supplementary information). In addition, the BECs for $\mathrm{Ba}$ and $\mathrm{Sr}$ are similar and close to the formal charge of +2 , demonstrating that these ions do not significantly change their covalency in the ferroelectric transition.

Our results point to a conventional ferroelectric mechanism in which polarization arises from off-centering of $d^{0} \mathrm{Nb}^{5+}$ ions in the $\mathrm{NbO}_{6}$ octahedra, which is in turn stabilized by the resulting enhanced $\mathrm{Nb}-\mathrm{O}$ covalency. Partial covalency is well known as the origin of ferroelectricity in perovskite oxides [34-36], and is classified as a second-order Jahn-Teller effect [4]. The charge densities we obtain for BN and SN (Figure $4 a-b)$ are visually similar to those found experimentally for the prototype ferroelectrics $\mathrm{BaTiO}_{3}$ and $\mathrm{PbTiO}_{3}$ [37], and the large BECs we find are close to previous calculations for $\mathrm{Nb}$ and $\mathrm{O}$ in $\mathrm{KNbO}_{3}$ [32]. The interpretation of large BECs is that additional covalency associated with the polar distortion occurs through electron transfer from $\mathrm{O} 2 p$ to the formally empty $\mathrm{Nb}$ $4 d$ orbitals, thereby enhancing the charge transfer compared to simple ionic displacement with nominal charges. The calculated $P_{z}$ (Figure $3 \mathrm{c}$ ) show that the polarization is robust with respect to cation-vacancy disorder, and that a significant polarization persists for all ten configurations for both compounds. This is also evident from the charge densities (Figure $4 a-b$ ) which demonstrate that the off-centering of $\mathrm{Nb}^{5+}$ is significant even for the ground state of SN in the SS[001] configuration, where the tilt-mode amplitude is large. Octahedral tilting in
TTBs is otherwise restricted by the connectivity in the $x y$ plane, which prevents tilting around the $z$ axis. Tilts around axes in the $x y$ plane are however possible, and often lead to a $\sqrt{2} \times \sqrt{2} \times 2$ supercell relative to the aristotype [10,38], in line with a recent group-theoretical analysis [39]. A $\sqrt{2} \times \sqrt{2} \times 2$ supercell was also reported for pure SN with TTB structure [40], although for intermediate SBN compositions, doubling of the $c$ parameter has to our knowledge not been reported.

It is generally accepted that chemical or structural disorder is necessary for relaxor properties [9], and both are possible in $\mathrm{SBN}$ due to $\mathrm{Sr}^{2+}, \mathrm{Ba}^{2+}$ and vacancies occupying the same sublattices. In disordered perovskite relaxors the two cations occupying the same sublattice are always aliovalent, examples being B-site disordered $\mathrm{PbMg}_{1 / 3} \mathrm{Nb}_{2 / 3} \mathrm{O}_{3}$ (PMN) [9, 41] and A-site disordered $\mathrm{Bi}_{1 / 2} \mathrm{Na}_{1 / 2} \mathrm{TiO}_{3}$ (BNT) $[18,42]$. In PMN and $\mathrm{BNT}$ the cation ratios are fixed by electroneutrality, whereas in SBN the A-site composition can be varied continuously and relaxor properties emerge gradually for higher Sr content. This points to a different relaxor mechanism for SBN than for the perovskites. We investigate the possible role of the A-site cations in SBN by displacing $\mathrm{Ba}$ and $\mathrm{Sr}$ away from their relaxed positions in the A2 channels and mapping the potential energy surface (PES) as shown in Figure 4c-d. The PES is flatter for $\mathrm{SN}$ than for $\mathrm{BN}$, which is explained by $\mathrm{Sr}^{2+}$ being smaller than $\mathrm{Ba}^{2+}$. Due to the restrictions imposed by the octahedral connectivity, and the fact that not all cation configurations in SBN produce tilted ground states, the TTB structure cannot compensate for this by octahedral tilting. Based on this, we suggest that $\mathrm{Sr}^{2+}$ is able to displace inside the A2 channels under application of an electric field, thereby producing an additional dielectric response which creates a diffuse phase transition for Sr-rich SBN. Sr preferentially occupies the A1 sites in SBN [19, 43], but for $x>0.40$, Sr must also occupy A 2 sites. For $x>0.50$, the amount of $\mathrm{Sr}$ on A2 is high enough for this displacement effect to be appreciable, and we propose that this induces relaxor behavior. The mechanism is different from perovskite relaxors in which cation ordering determines the type of dielectric response [44]. The properties demonstrated here should apply also to other TTB oxides with [001] polarization.

In conclusion, we have shown that the ferroelectric polarization in $\mathrm{BN}$ and $\mathrm{SN}$ with TTB structure originates from a single polar instability. Charge densities and Born effective charges point to a conventional ferroelectric mechanism in the form of a second-order Jahn-Teller effect acting on the $d^{0} \mathrm{Nb}^{5+}$ cations. A second unstable mode leads to tilting of the $\mathrm{NbO}_{6}$ octahedra in the $x y$ plane. The ferroelectric polarization is robust with respect to cation-vacancy disorder, even when the ground state involves significant octahedral tilting. We suggest that this ferroelectric mechanism is present for all compositions of $\mathrm{SBN}$, and that relaxor behavior emerges as an additional contribution for high $\mathrm{Sr}$ content due to the smaller $\mathrm{Sr}^{2+}$ displacing inside the pentagonal channels.

The Research Council of Norway (NFR project no. 209337) and The Faculty of Natural Sciences and Technology, Norwegian University of Science and Technology (NTNU) are 
acknowledged for financial support. Computational resources were provided by ETH Zurich and NOTUR (The Norwegian Metacenter for High Performance Computing) through the project NN9264K.

* Corresponding author: tor.grande@ntnu.no

[1] K. Momma and F. Izumi, J. Appl. Cryst. 44, 1272 (2011).

[2] A. M. Glass, J. Appl. Phys. 40, 4699 (1969).

[3] G. Goodman, J. Am. Ceram. Soc. 36, 368 (1953).

[4] P. S. Halasyamani and K. R. Poeppelmeier, Chem. Mater. 10, 2753 (1998).

[5] N. A. Hill, J. Phys. Chem. B 104, 6694 (2000).

[6] M. H. Francombe, Acta Crystallogr. 9, 683 (1956).

[7] M. G. Sahini, T. Grande, B. Fraygola, A. Biancoli, D. Damjanovic, and N. Setter, J. Am. Ceram. Soc. 97, 220 (2014).

[8] G. H. Olsen, M. H. Sørby, B. C. Hauback, S. M. Selbach, and T. Grande, Inorg. Chem. 53, 9715 (2014).

[9] L. E. Cross, Ferroelectrics 76, 241 (1987).

[10] P. Labbe, Key Eng. Mater. 68, 293 (1992).

[11] X. Zhu, M. Fu, M. C. Stennett, P. M. Vilarinho, I. Levin, C. A. Randall, J. Gardner, F. D. Morrison, and I. M. Reaney, Chem. Mater. 27, 3250 (2015).

[12] P. B. Jamieson, S. C. Abrahams, and J. L. Bernstein, J. Chem. Phys. 48, 5048 (1968).

[13] P. B. Jamieson, S. C. Abrahams, and J. L. Bernstein, J. Chem. Phys. 50, 4352 (1969).

[14] S. C. Abrahams, P. B. Jamieson, and J. L. Bernstein, J. Chem. Phys. 54, 2355 (1971).

[15] M. H. Francombe, Acta Crystallogr. 13, 131 (1960).

[16] K. L. Keester, R. R. Neurgaonkar, T. C. Lim, and E. J. Staples, Mater. Res. Bull. 15, 821 (1980).

[17] J. Ravez and A. Simon, J. Solid State Chem. 162, 260 (2001).

[18] V. V. Shvartsman and D. C. Lupascu, J. Am. Ceram. Soc. 95, 1 (2012).

[19] G. H. Olsen, S. M. Selbach, and T. Grande, Phys. Chem. Chem. Phys. 17, 30343 (2015)
[20] H. T. Stokes and D. M. Hatch, J. Appl. Crystallogr. 38, 237 (2005)

[21] G. Kresse and J. Furthmüller, Phys. Rev. B 54, 11169 (1996).

[22] J. P. Perdew, A. Ruzsinszky, G. I. Csonka, O. A. Vydrov, G. E. Scuseria, L. A. Constantin, X. Zhou, and K. Burke, Phys. Rev. Lett. 100, 136406 (2008).

[23] G. Kresse and D. Joubert, Phys. Rev. B 59, 11 (1999).

[24] H. J. Monkhorst and J. D. Pack, Phys. Rev. B 13, 5188 (1976).

[25] A. Togo and I. Tanaka, Scripta Mater. 108, 1 (2015).

[26] A. Togo, F. Oba, and I. Tanaka, Phys. Rev. B 78, 134106 (2008).

[27] R. King-Smith and D. Vanderbilt, Phys. Rev. B 47, 1651 (1993).

[28] R. Resta, Rev. Mod. Phys. 66, 899 (1994).

[29] N. A. Spaldin, J. Solid State Chem. 195, 2 (2012).

[30] M. T. Dove, Am. Mineral. 82, 213 (1997).

[31] D. Orobengoa, C. Capillas, M. I. Aroyo, and J. M. Perez-Mato, J. Appl. Crystallogr. 42, 820 (2009).

[32] P. Ghosez, J.-P. Michenaud, and X. Gonze, Phys. Rev. B 58, 6224 (1998).

[33] F. Detraux, P. Ghosez, and X. Gonze, Phys. Rev. B 56, 983 (1997).

[34] H. D. Megaw, Acta Crystallogr. 5, 739 (1952).

[35] I. B. Bersuker, Phys. Lett. 20, 589 (1966).

[36] R. Cohen, Nature 358, 136 (1992).

[37] Y. Kuroiwa, S. Aoyagi, A. Sawada, J. Harada, E. Nishibori, M. Takata, and M. Sakata, Phys. Rev. Lett. 87, 217601 (2001).

[38] B. A. Scott, E. A. Giess, G. Burns, and D. F. O'Kane, Mater. Res. Bull. 3, 831 (1968).

[39] T. A. Whittle, S. Schmid, and C. J. Howard, Acta Crystallogr. B 71, 342 (2015).

[40] H. Brusset, M. Gillier-Pandraud, and S. D. Voliotis, Mater. Res. Bull. 6, 5 (1971).

[41] S. Tinte, B. P. Burton, E. Cockayne, and U. V. Waghmare, Phys. Rev. Lett. 97, 137601 (2006).

[42] M. Gröting, S. Hayn, and K. Albe, J. Solid State Chem. 184, 2041 (2011).

[43] S. Podlozhenov, H. A. Graetsch, J. Schneider, M. Ulex, M. Wöhlecke, and K. Betzler, Acta Crystallogr. B 62, 960 (2006).

[44] N. Setter and L. E. Cross, J. Appl. Phys. 51, 4356 (1980). 